\section{La télomérase de la levure subit une cure de rajeunissement}

Nancy Laterreur, Raymund J. Wellinger
Département de microbiologie et infectiologie, Faculté de médecine et des sciences de la santé, Université de Sherbrooke, 3201 Rue Jean Mignault Sherbrooke, Québec, JlE 4K8, Canada.

raymund.wellinger@usherbrooke.ca

télomérase, mais sa fonction précise reste inconnue [10].

Nos connaissances de l'organisation protéique de la télomérase, chez la levure, sont demeurées inchangées ces quinze dernières années. Cependant, de par sa très grande taille et sa structure, il était raisonnable de penser que I'ARN TIcl pouvait recruter d'autres protéines. La télomérase est réactivée dans plus de $90 \%$ des cancers. La découverte de nouveaux acteurs interagissant avec l'ARN pourrait donc contribuer au développement de nouvelles cibles thérapeutiques. Considérant ces données, nous avons entrepris la purification de la télomérase de Saccharomyces cerevisiae, afin d'enrichir notre connaissance de ce complexe enzymatique.

La purification de la télomérase a reposé sur le système MS2 [11]: dix tigesboucles provenant de I'ARN génomique du bactériophage MS2 ont été insérées à l'extrémité 3' de l'ARN TIcl. La protéine de capside du phage MS2, qui reconnaît spécifiquement ces tiges-boucles, fusionnée avec la protéine A de la bactérie Staphylococcus aureus, appelée ProA, a été exprimée. II est ainsi possible de purifier le complexe entier grâce à cette étiquette ProA, qui lie spécifiquement les immunoglobulines (Figure IB). Après immobilisation sur des billes couplées aux immunoglobulines, l'extrait protéique est préparé pour la spectrométrie de masse (MS). En parallèle, un extrait de protéines dans lequel TIcl ne possède pas les tiges-boucles de MS2, a également été traité comme contrôle négatif, afin d'identifier les protéines qui seraient liées non-spécifiquement. 


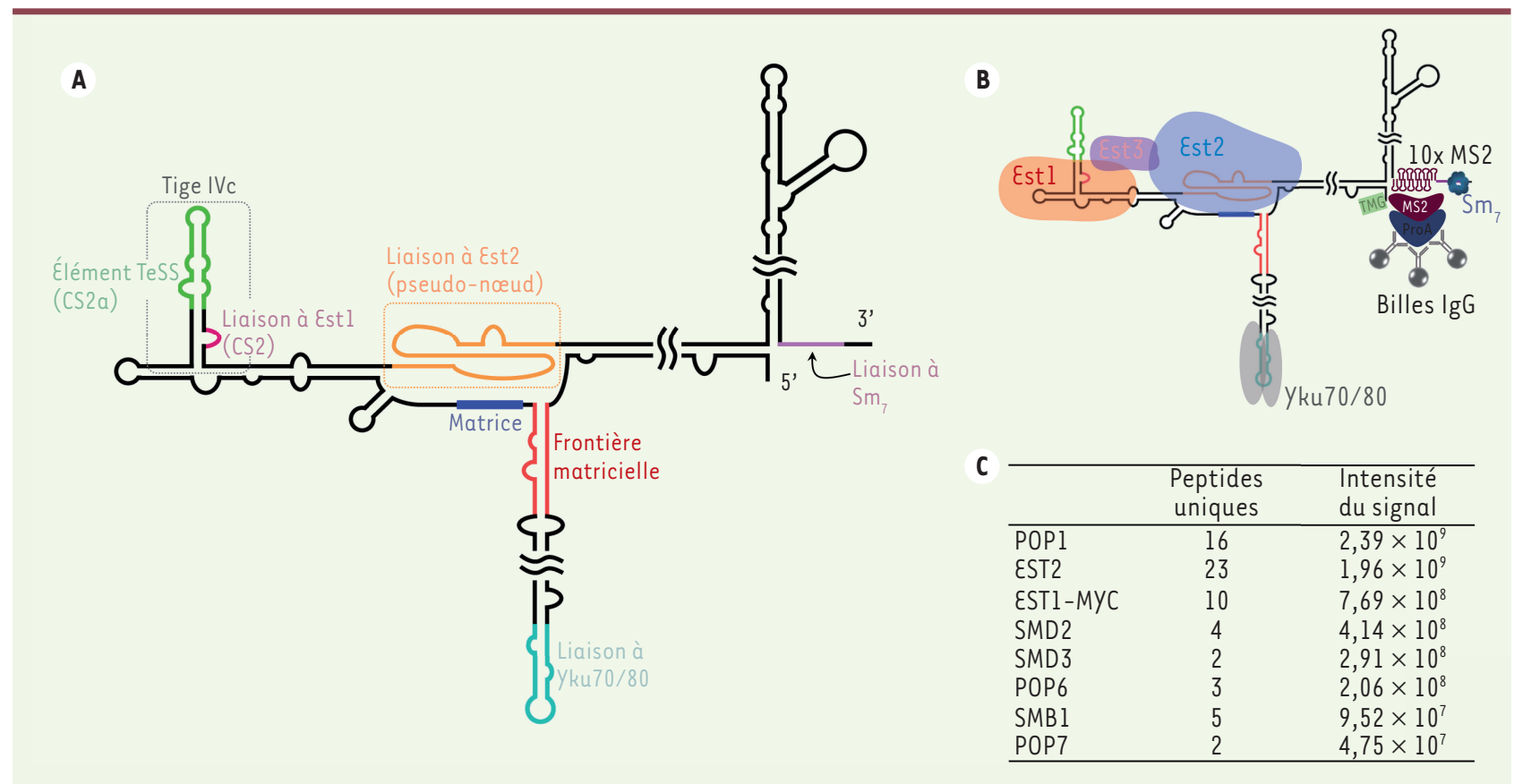

Figure 1. L'ARN Tlcl est utilisé comme appât pour la purification de la télomérase, le complexe est ensuite analysé par spectrométrie de masse. A. Structure secondaire générale de TIcl. On note, selon les couleurs, les régions importantes de l'ARN qui sont connues pour lier des protéines. Une exception : l'élément TeSS/CS2a (telomerase-stimulating structure/ conserved sequence $2 a$ ) de la tige IVc dont la contribution au complexe demeurait à déterminer. B. Schéma de la purification de la télomérase via l'ARN TIcl. Dix tiges-boucles provenant de l'ARN génomique du bactériophage MS2 sont insérées à l'extrémité 3' de l'ARN pour ainsi l'immunoprécipiter et entraîner les protéines qui y sont attachées. La protéine capside du phage MS2 (fusionnée à l'étiquette ProA) interagit avec les tiges-boucles MS2. Des billes couvertes d'immunoglobulines $G$ (IgG) reconnaissent l'étiquette ProA et permettent l'immunopurification. C. Tableau présentant les 8 meilleurs candidats identifiés au cours de 3 expériences indépendantes de purification et d'analyse par spectrométrie de masse. La liste est organisée par ordre d'intensité du signal obtenu et le nombre de peptides retrouvés pour chacune d'entre elles est indiqué.

Peu de candidats, mais une agréable coïncidence

Les résultats initiaux de MS ont identifié peu de candidats (Figure IC). Parmi les plus abondants, se trouvaient des protéines connues pour lier l'ARN de la télomérase, comme Estl, Est2 et certaines protéines du complexe $\mathrm{Sm}_{7}$. Nous étions donc très confiants sur ces résultats, d'autant que ces protéines étaient identifiées de façon spécifique. L'analyse a également révélé d'autres candidats, inconnus comme partenaires de la télomérase : les ribonucléases Popl (processing of precursor 1), Pop6 et Pop7 (Figure 1C).

Les protéines Popl, Pop6 et Pop7 sont des sous-unités des complexes ribonucléases (RNase) P/MRP, chez la levure. Ces deux complexes essentiels sont hautement conservés et sont responsables du traitement des ARN de transfert (ARNt), des ARN ribosomaux (ARNr) et de certains ARN messagers (ARNm) [12]. Les deux complexes ribonucléases, P/MRP, contiennent, tout comme la télomérase, plusieurs sous-unités protéiques qui sont assemblées autour d'un ARN non-codant (nommés respectivement $\mathrm{Nmel}$ pour la RNase MRP et Rprl pour la RNase P). L'analyse de la liaison de Popl, Pop6 et Pop7 aux ARN permet de remarquer que Pop6 et Pop7 forment un hétérodimère qui lie une tigeboucle particulière, présente dans les deux ARN ( $\mathrm{Nmel}$ et Rprl) et nommée domaine P3. La protéine Popl, elle, est recrutée, ensuite, pour stabiliser le complexe entier. Elle entraînera la liai- son des autres protéines partenaires des ribonucléases P/MRP [13, 14].

Une analyse précise de la structure de I'ARN TIcl montre que la région distale de la tige IVc, qui contient la séquence CS2a et le domaine TeSS (telomerase-stimulating structure), présente de fortes homologies avec le domaine P3 de Nmel et Rprl (Figure 2A). En fait, les structures générales sont identiques et la séquence de la boucle principale est hautement conservée. Pour Nmel et Rprl, le contact avec l'hétérodimère Pop6/Pop7 est assuré par la portion $\mathrm{N}$-terminale (nucléotides 30 à 40 ) de la boucle du domaine P3 [12]. Pour TIcl, cette même région est essentielle à l'activité de la télomérase, sans que soit toutefois précisé s'il s'agit d'un site de liaison à des protéines [10]. 


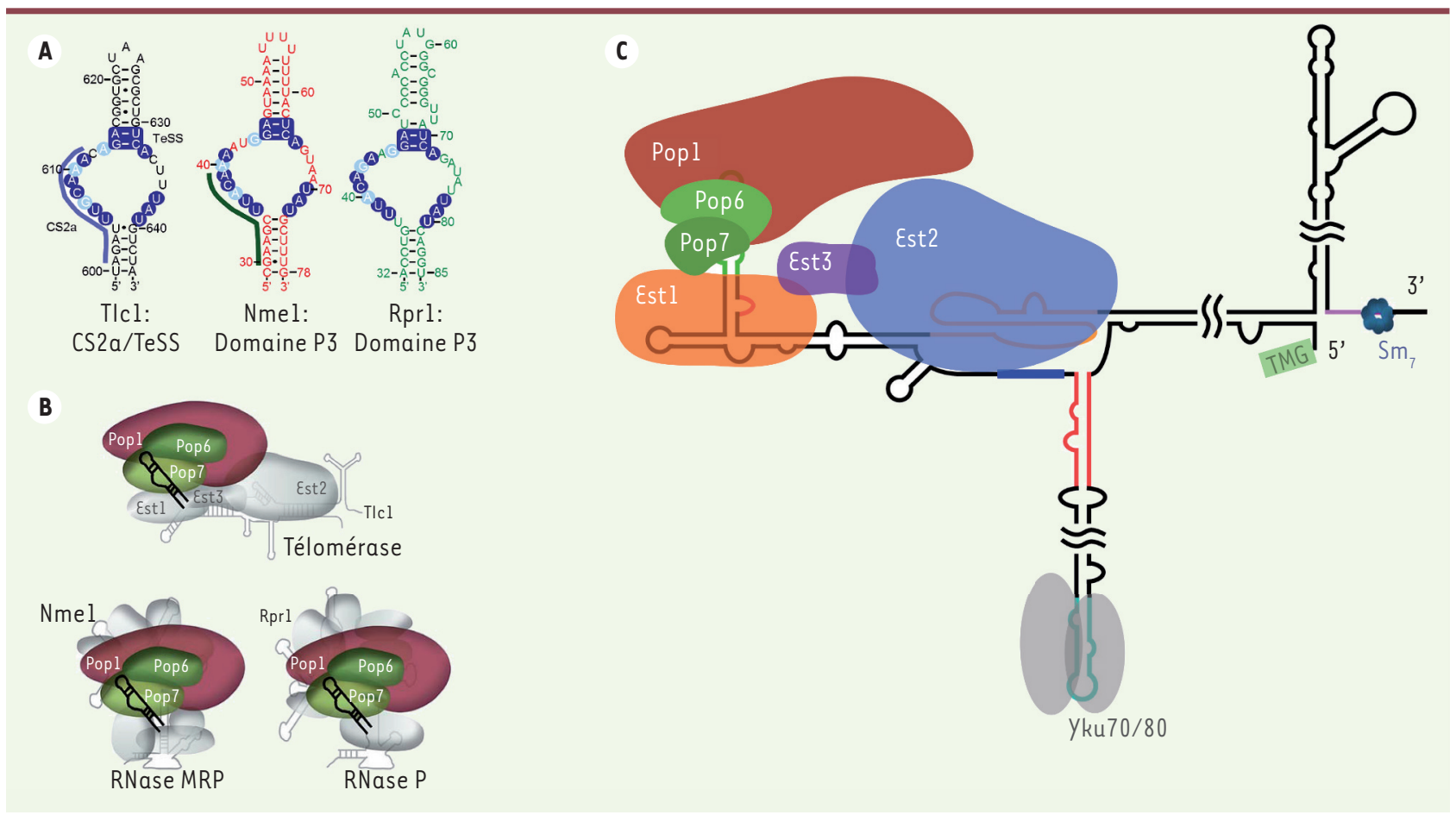

Figure 2. Le domaine P3 de Tlc1 possède les mêmes fonctions que celui des ARN Nmel et Rprl des ribonucléases MRP/P : il lie l'hétérodimère Pop6/ Pop7 pour ensuite recruter la protéine Pop1. A. Représentation de la portion distale de la tige IVc : le domaine TeSS (telomerase-stimulating structure) et la séquence CS2a (conserved sequence $2 a$ ) possèdent de fortes ressemblances avec les domaines P3 des ARN Nmel et Rprl. Les nucléotides (nt) identiques sont encerclés en bleu foncé. Les purines et pyrimidines conservées sont encerclées en bleu pâle. Le trait bleu à gauche de Tlcl représente la portion essentielle à l'activité de la télomérase, l'élément CS2a. Le trait vert foncé à gauche de Nmel représente les nucléotides protégés par la liaison de Pop6/Pop7. B. Les ARN de la télomérase (TIcl), la ribonucléase (RNase) MRP (Nmel) et la RNase P (Rprl) ont un élément commun : le domaine P3. Celui-ci est parfaitement interchangeable entre les trois ARN. C. Nouveau modèle de la télomérase chez la levure Saccharomyces cerevisiae. Le domaine P3 de Tlcl recrute l'hétérodimère Pop6/Pop7. La protéine Popl se lie, par la suite, pour stabiliser le complexe de la télomérase formé des protéines Estl (ever shorter telomere 1), Est2 et Est3.

\section{Tlcl possède un domaine $\mathrm{P3}$} liant les protéines Pop6/Pop7

Afin d'examiner si la portion distale de la tige IVc de TIcl présente les mêmes fonctionnalités que le domaine $\mathrm{P} 3 \mathrm{de} \mathrm{Nmel}$ et Rprl, nous avons échangé les sous-domaines entre les différents ARN. La région de TIcl correspondant au domaine P3 a ainsi été insérée à la place du domaine P3 de Nmel, et les domaines P3 de Nmel ou de Rprl ont remplacé le possible domaine P3 de TIcl. Dans le premier cas, la RNase MRP (qui comporte l'ARN Nmel), essentielle chez la levure, une non-fonctionnalité de la portion dérivée de Tlcl devrait se traduire par la mort des cellules. Les résultats obtenus montrent, en fait, une complète interchangeabilité entre le domaine P3 de Tlcl et celui de Nmel.
Inversement, les domaines P3 de Nmel et de Rprl assurent une fonctionnalité totale de la télomérase lorsqu'ils remplacent la structure distale de la tige IVc de T|cl, qui est essentielle à l'activité de l'enzyme télomérase (Figure 2B).

Afin de démontrer que le domaine $\mathrm{P} 3$ de TIcl lie les protéines Pop6/Pop7 comme c'est le cas pour les RNases, des expériences de co-immunoprécipitation ont été réalisées. Dans des souches de levure exprimant une étiquette de reconnaissance sur une protéine du complexe $\mathrm{Sm}_{7}$ (Smel) ainsi que sur les protéines Pop6 ou Pop7, nous avons pu démontrer qu'en l'absence du domaine TeSS/P3, l'ARN TIcl peut être co-immunoprécipité avec Smel, mais qu'il n'entraîne pas les protéines Pop6 ou Pop7. Par ailleurs, l'ajout de la protéine Popl, sous forme recombinante, à une réaction contenant $T \mid c l, \varepsilon s t 2 p$ et Pop6/Pop7, dans des expériences de reconstitution in vitro, augmente significativement l'activité de la télomérase. La protéine Popl serait donc nécessaire pour stabiliser le complexe enzymatique après la liaison de Pop6/Pop7.

De nouvelles protéines entrent dans la constitution du complexe de la télomérase chez Saccharomyces cerevisiae (Figure 2C). II est donc nécessaire d'actualiser l'ancien modèle et de le redessiner totalement. Ces découvertes soulèvent aussi plusieurs questions importantes. Est-ce un hasard si des complexes RNP considérés comme très anciens en termes d'évolution, partagent un tel mode de fonctionnement? 
Pourquoi la télomérase nécessite-t-elle les protéines Pop6/Pop7 et Popl pour son fonctionnement alors que la télomérase et les ribonucléases jouent des rôles aussi différents au sein de la cellule? Quelle est la séquence des événements lors de l'assemblage du complexe ? Et, de façon plus large, l'association des protéines Pop à la télomérase est-elle conservée jusqu'aux vertébrés, et même les mammifères? Cette découverte soulève beaucoup d'enthousiasme : l'identification de nouvelles protéines dans un complexe enzymatique aussi important que la télomérase pourrait permettre de mieux comprendre son mode d'action et, éventuellement de découvrir de nouvelles cibles thérapeutiques contre le cancer! $\diamond$

A rejuvenation for yeast telomerase

\section{LIENS D'INTÉRÊT}

Les auteurs déclarent n'avoir aucun lien d'intérêt concernant les données publiées dans cet article.

\section{RÉFÉRENCES}

1. Gallardo F, Chantrand P. Biogenèse de la télomérase : un long voyage jusqu'au bout des chromosomes. Med Sci (Paris) $2009 ; 25: 232-4$.

2. Lemieux $B$, Laterreur $N$, Perederina $A$, et al. Active yeast telomerase shares subunits with ribonucleoproteins RNase P and RNase MRP. Cell 2016; 165 : 1171-81.

3. Wellinger RJ, Zakian VA. Everything you ever wanted to know about Saccharomyces cerevisiae telomeres: beginning to end. Genetics 2012 ; 191 : 1073-105.

4. Forstemann K, Lingner J. Telomerase limits the extent of base pairing between template RNA and telomeric DNA. EMBO Rep 2005 ; 6 : 361-6.

5. Zappulla DC, Cech TR. Yeast telomerase RNA: A flexible scaffold for protein subunits. Proc Natl Acad Sci USA $2004 ; 101: 10024-9$.

6. Dandjinou AT, Levesque $\mathrm{N}$, Larose $\mathrm{S}$, et al. A phylogenetically based secondary structure for the yeast telomerase RNA. Curr Biol $2004 ; 14$ : 1148-58.
7. Zappulla DC, Cech TR. RNA as a flexible scaffold for proteins: yeast telomerase and beyond. Cold Spring Harb Symp Quant Biol 2006 ; 71 : 217-24.

8. Gunisova S, Elboher $\varepsilon$, Nosek J, et al. Identification and comparative analysis of telomerase RNAs from Candida species reveal conservation of functional elements. RNA 2009 ; 15 : 546-59.

9. Seto AG, Livengood AJ, Tzfati Y, et al. A bulged stem tethers Estlp to telomerase RNA in budding yeast. Genes Dev $2002 ; 16: 2800-12$.

10. Laterreur $\mathrm{N}$, Eschbach SH, Lafontaine DA, Wellinger RJ. A new telomerase RNA element that is critical for telomere elongation. Nucleic Acids Res 2013 ; 41 : 7713-24.

11. Gallardo F, Laterreur N, Cusanelli $\varepsilon$, et al. Live cell imaging of telomerase RNA dynamics reveals cell cycle-dependent clustering of telomerase at elongating telomeres. Mol Cell 2011 ; $44: 819-27$.

12. Esakova 0 , Krasilnikov AS. Of proteins and RNA: the RNase P/MRP family. RNA $2010 ; 16$ : 1725-47.

13. Perederina $A$, Esakova 0 , Quan $C$, et al. Eukaryotic ribonucleases P/MRP: the crystal structure of the P3 domain. EMBO J 2010 ; 29 : 761-9.

14. Perederina $A$, Esakova 0 , Koc $H$, et al. Specific binding of a Pop6/Pop7 heterodimer to the P3 stem of the yeast RNase MRP and RNase P RNAs. RNA 2007 ; 13 : 1648-55.

B ertrand Jordan a participé à l'extraordinaire aventure de la biologie moléculaire, encore balbutiante dans les années 1960, mais qui a complètement révolutionné les sciences du vivant au cours des décennies suivantes.

L'ADN, quasiment inaccessible au début de cette période, intervient maintenant dans d'innombrables recherches, de l'écologie à l'anthropologie, sans oublier bien sûr la médecine dont il fait désormais partie intégrante. Nous pouvons aujourd'hui lire intégralement

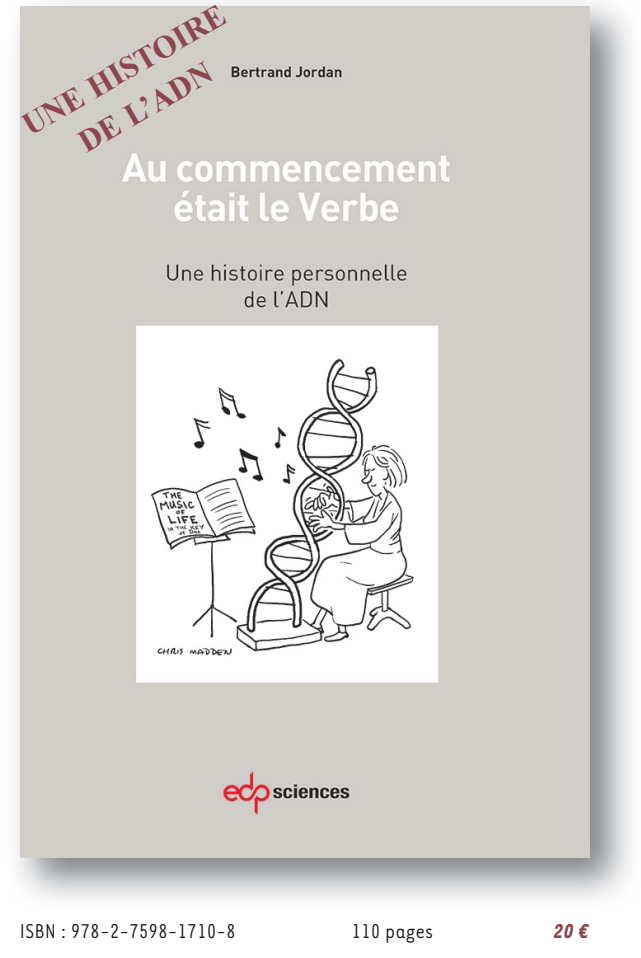

I'ADN d'une personne en quelques heures, et en tirer de précieuses informations pour la prévention et le traitement de nombreuses maladies - et nous n'en sommes qu'au début de cette nouvelle médecine!

Acteur mais aussi témoin de ces avancées, Bertrand Jordan fait ici un récit très personnel et sans langue de bois de sa vie avec l'ADN. Ce livre ne prétend pas être une histoire complète de la biologie moléculaire, mais il illustre son développement, révélant parfois le «dessous des cartes» grâce aux expériences vécues par son auteur.

$3<$

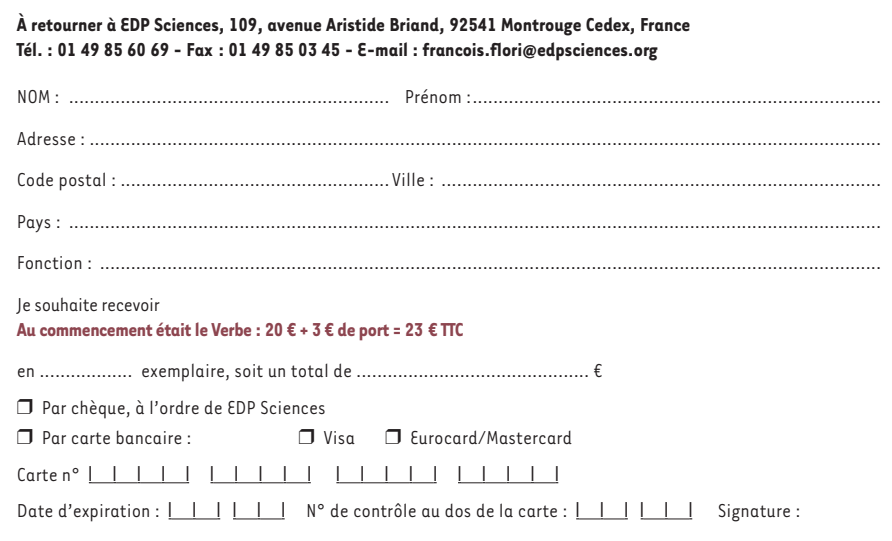

